\title{
Possible ring material around centaur (2060) Chiron $\star$
}

\author{
J. L. Ortiz ${ }^{1}$, R. Duffard ${ }^{1}$, N. Pinilla-Alonso ${ }^{2}$, A. Alvarez-Candal ${ }^{3}$, P. Santos-Sanz ${ }^{1}$, N. Morales ${ }^{1}$, \\ E. Fernández-Valenzuela ${ }^{1}$, J. Licandro ${ }^{4,5}$, A. Campo Bagatin ${ }^{6}$, and A. Thirouin ${ }^{1,7}$
}

\author{
1 Instituto de Astrofísica de Andalucía, CSIC, Apt 3004, 18080 Granada, Spain \\ e-mail: duffard@iaa.es \\ 2 Department of Earth and Planetary Sciences, University of Tennessee, Knoxville, 37996 TN, USA \\ 3 Observatório Nacional, Rua General José Cristino 77, 20921-400 Rio de Janeiro, Brazil \\ ${ }^{4}$ Instituto de Astrofísica de Canarias, c/Vía Lactea s/n, 38200 La Laguna, Tenerife, Spain \\ 5 Departamento de Astrofísica, Universidad de La Laguna (ULL), 38205 La Laguna, Tenerife, Spain \\ 6 Departamento de Física, Ingeniería de Sistemas y Teoría de la Señal, Universidad de Alicante, Carretera San Vicente del Raspeig s/n, \\ 03690 San Vicente del Raspeig, Alicante, Spain \\ 7 Lowell Observatory, 1400 W Mars Hill Rd, Flagstaff, 86001 Arizona, USA
}

Received 24 June 2014 / Accepted 12 January 2015

\section{ABSTRACT}

\begin{abstract}
We propose that several short-duration events observed in past stellar occultations by Chiron were produced by ring material. Some similarities between these events and the characteristics of Chariklo's rings could indicate common mechanisms around centaurs. From a reanalysis of the stellar occultation data in the literature, we determined two possible orientations of the pole of Chiron's rings, with ecliptic coordinates $\lambda=(352 \pm 10)^{\circ}, \beta=(37 \pm 10)^{\circ}$ or $\lambda=(144 \pm 10)^{\circ}, \beta=(24 \pm 10)^{\circ}$. The mean radius of the rings is $(324 \pm 10) \mathrm{km}$. One can use the rotational lightcurve amplitude of Chiron at different epochs to distinguish between the two solutions for the pole. Both solutions imply a lower lightcurve amplitude in 2013 than in 1988, when the rotational lightcurve was first determined. We derived Chiron's rotational lightcurve in 2013 from observations at the $1.23 \mathrm{~m}$ CAHA telescope, and indeed its amplitude was smaller than in 1988. We also present a rotational lightcurve in 2000 from images taken at the CASLEO $2.15 \mathrm{~m}$ telescope that is consistent with our predictions. Out of the two poles, the $\lambda=(144 \pm 10)^{\circ}, \beta=(24 \pm 10)^{\circ}$ solution provides a better match to a compilation of rotational lightcurve amplitudes from the literature and those presented here. We also show that using this preferred pole orientation, Chiron's long-term brightness variations are compatible with a simple model that incorporates the changing brightness of the rings while the tilt angle with respect to the Earth is changing with time. Also, the variability of the water ice band in Chiron's spectra as seen in the literature can be explained to a large degree by an icy ring system whose tilt angle changes with time and whose composition includes water ice, analogously to the case of Chariklo. We present several possible formation scenarios for the rings from qualitative points of view and speculate on why rings might be common in centaurs. We also speculate on whether the known bimodal color distribution of the centaurs could be due to centaurs with rings and centaurs without rings.
\end{abstract}

Key words. minor planets, asteroids: individual: (2060) Chiron - planets and satellites: rings - techniques: imaging spectroscopy occultations

\section{Introduction}

The object (2060) Chiron (formerly 1977 UB) was the first of a new class of objects discovered in our solar system, the so-called "centaurs". These objects orbit the Sun at distances between Jupiter and Neptune. Soon after the discovery of Chiron several decades ago, this centaur was reported as showing cometary-like activity (Meech \& Belton 1989). It had several brightness outbursts and developed coma, like a comet (Luu \& Jewitt 1990). For this reason Chiron is listed as a solar system object with a dual designation: comet 95P/Chiron and minor planet (2060) Chiron.

Because Chiron is a very interesting body, it has been extensively observed and studied with many techniques. From short-term photometry studies, rotational lightcurves have been determined for Chiron at different epochs (Bus et al. 1989; Marcialis \& Buratti 1993; Lazzaro et al. 1997), showing that it has an elongated shape that causes a double peaked lightcurve.

* Table 1 is only available at the CDS via anonymous ftp to cdsarc.u-strasbg.fr (130.79.128.5) or via http://cdsarc.u-strasbg.fr/viz-bin/qcat?J/A+A/576/A18
A very high accuracy rotational period of $5.917813 \mathrm{~h}$ has been determined (Marcialis \& Buratti 1993) and also some constraints on the shape of the body obtained (e.g., Groussin et al. 2004). Spectroscopically, Chiron has been shown to possess water ice features in the near infrared, whose exact widths seem to vary according to different authors who observed in different epochs (Foster et al. 1999; Luu et al. 2000; Romon-Martin et al. 2003). These features even disappeared in 2001 (Romon-Martin et al. 2003). From thermal infrared observations and modeling, the equivalent diameter of Chiron has been shown to be around $180 \mathrm{~km}$, although considerable scatter has been found (e.g., Groussin et al. 2004).

After the recent and surprising discovery by stellar occultation observations that another centaur, Chariklo, has two dense rings (Braga-Ribas et al. 2014), we wondered whether other centaurs could have rings. Because the rings of Chariklo are so close to the main body and subtend a tiny angle of around 0.08 arcsec, direct imaging detection of rings is challenging with current technology, so stellar occultations are the best means of detecting rings around small bodies. Apart from Chariklo, the only centaur for which stellar occultations have been successfully 
recorded in the past is Chiron, and curiously, the lightcurves of these occultations showed rapid secondary dimmings, which were interpreted as comet-like dust jets from the nucleus in 1993 and 1994 occultations (Elliot et al. 1995; Bus et al. 1996) and as symmetric jet-like features (Ruprecht 2013; Ruprecht et al. 2013 ) in a 2011 occultation. Jets seemed to be a natural explanation given that Chiron had shown cometary activity in the past. However, the width of the two features reported by Ruprecht (2013) were 3 and $7 \mathrm{~km}$, respectively, separated by a distance of 10 to $14 \mathrm{~km}$, which are nearly identical to the $6.6 \mathrm{~km}$ and $3.4 \mathrm{~km}$ widths and $9 \mathrm{~km}$ separation of the rings in Chariklo as reported by Braga-Ribas et al. (2014). Our interpretation of the sharp events in the Chiron 2011 occultation lightcurves is that these features were caused by two rings with similar properties to those recently reported for Chariklo's rings by Braga-Ribas et al. (2014). Here we present such an interpretation.

In Sect. 2 of this paper we present a reanalysis of the occultation lightcurves available in the literature, from which we derive two possible pole orientations for the proposed ring system. In Sect. 3, we show that the change in Chiron's water ice spectral features with time is consistent with the proposed rings. In Sect. 4 we show that the ring system can account for a large portion of the overall long-term photometric behavior of Chiron. In Sect. 5 we present two new rotational lightcurves that we obtained from the CAHA $1.23 \mathrm{~m}$ telescope in 2013 and from the CASLEO $2.15 \mathrm{~m}$ telescope in 2000, and we show that the observed changes in the amplitude of the rotational lightcurves are consistent with expectations based on the preferred orientation of the ring system. In Sect. 6 we show more indications of the ring system.

In the last section we discuss our results and speculate on why Chiron, the second largest centaur after Chariklo (Duffard et al. 2014b; Fornasier et al. 2013), can also have rings and whether rings can be common in centaurs and other outer solar system bodies. Along with this, we present some scenarios of ring formation from qualitative points of view and present different ideas to can explore in the future.

\section{Reanalysis of stellar occultations}

Three stellar occultations by Chiron have been reported since its discovery. The first stellar occultation was on November 7 , 1993, at around 13:16 UT, and the results were published in Bus et al. (1996), where all the pertinent details are given. We briefly mention that the magnitude of the star that was occulted was 14 and that the telescopes used were all smaller than $0.6 \mathrm{~m}$, except for one. Another stellar occultation was observed in 1994 on March 9 at around 23:29 UT, although the findings associated with this occultation were reported earlier than for the 1993 event (Elliot et al. 1995). The magnitude of the occulted star was 11.95 , so brighter than the one that was occulted in 1993, and the telescopes used were larger than in 1993, but only one occultation chord was obtained. On November 29, 2011 at around 8:16 UT another occultation by Chiron was observed and reported by Ruprecht (2013) and Ruprecht et al. (2013). In this case the occulted star was around magnitude 14.8, but the two telescopes involved in the observations were large, $2 \mathrm{~m}$ and $3 \mathrm{~m}$ in diameter, and provided the best data set.

In the Elliot et al. (1995) paper, the first paper on an occultation by Chiron, two clear short-duration drops in the lightcurves were reported and a few other longer lasting shallow events were reported, too. They were all attributed to dust jets like those of comets, which seemed a reasonable explanation because of the known cometary-like behavior of Chiron and also because symmetrical features with respect to the central body were not observed. That paper perhaps influenced the conclusions of the subsequent Bus et al. (1996) paper and the interpretation by Ruprecht (2013) and Ruprecht et al. (2013) of the occultation in 2011, which is the event that shows the most compelling evidence for a ring system, so those results will be discussed first.

\subsection{Stellar occultation in 2011}

Two datasets were obtained in this occultation, one from the NASA 3 m InfraRed Telescope Facility (IRTF) and another from the $2 \mathrm{~m}$ Faulkes telescope. Both datasets are reported in Ruprecht (2013) and Ruprecht et al. (2013). The data obtained with the NASA-IRTF telescope on Mauna Kea clearly show not only the occultation of Chiron but also two short symmetrical lightcurve drops prior to and after the occultation of the main body. (The symmetry is with respect to the center of Chiron's occultation.) The drops were at $\sim 300 \mathrm{~km}$ east and west of the center of the body occultation. The other dataset, obtained from the $2 \mathrm{~m}$ Faulkes telescope at Haleakala on Maui, provided a very high time-resolution $(0.2 \mathrm{~s})$, high signal-to-noise lightcurve, which was higher than what is usually achieved in stellar occultations by outer solar system bodies. That lightcurve does not show the occultation of Chiron's main body, but it shows two pairs of very sharp drops in the star's light.

All this is clearly illustrated in Figs. $2-2$ and $2-3$ of Ruprecht (2013). The lightcurve from the Faulkes telescope is very similar to the ones caused by Chariklo's rings as observed from the Danish $1.5 \mathrm{~m}$ telescope (Braga-Ribas et al. 2014). The width of the two features reported by Ruprecht (2013) were $3 \mathrm{~km}$ and $7 \mathrm{~km}$, respectively, separated by a distance of 10 to $14 \mathrm{~km}$, which are nearly identical to the $6.6 \mathrm{~km}$ and $3.4 \mathrm{~km}$ widths and $9 \mathrm{~km}$ separation of the rings in Chariklo as reported by Braga-Ribas et al. (2014). Also, the optical depths of the extinction features were very similar, 0.4 and 0.05 for Chariklo rings and 0.7 to 1 for Chiron. This similarity is remarkable. The only exception to the similarities of the $2 \mathrm{~m}$ Faulkes telescope dataset on the occultation by Chiron compared to the $1.5 \mathrm{~m}$ Danish telescope dataset on the occultation by Chariklo is that the occultation of the primary body was not detected in the Faulkes data, whereas it was in the $1.5 \mathrm{~m}$ Danish telescope lightcurve, but this difference has no implication on the detection of the rings. A double-ring system with a gap might possibly be related to the presence of an unknown shepherd satellite, as we hypothesize in the discussion section.

Using the timing information that can be obtained from the plots in Figs. 2, 3 of Ruprecht (2013) and using the 7 s offset that the authors applied to the Faulkes lightcurve (as explained in Ruprecht 2013, they applied that shift in order to align the occultation midpoint with the one seen in the IRTF data) we can derive approximate times for the disappearance of the rings as seen from the Faulkes $2 \mathrm{~m}$ telescope in Haleakala and from the IRTF telescope at Mauna Kea. A double-ring system is not resolved in the IRTF dataset because of its low time resolution ( $2 \mathrm{~s}$ ), so the times derived from the plot correspond to the middle of the occultation time of the two proposed rings. For the Faulkes data we have taken the mean time of the occultations of the two proposed rings so that we have something that can be compared to the data derived from the IRTF.

Because we know the coordinates of the two observing sites, we can project the secondary events in the plane of the sky. We can also plot the chord that corresponds to the body detection observed from the IRTF (but not detected from the $2 \mathrm{~m}$ Faulkes telescope). An infinite set of ellipses corresponding to a ring 


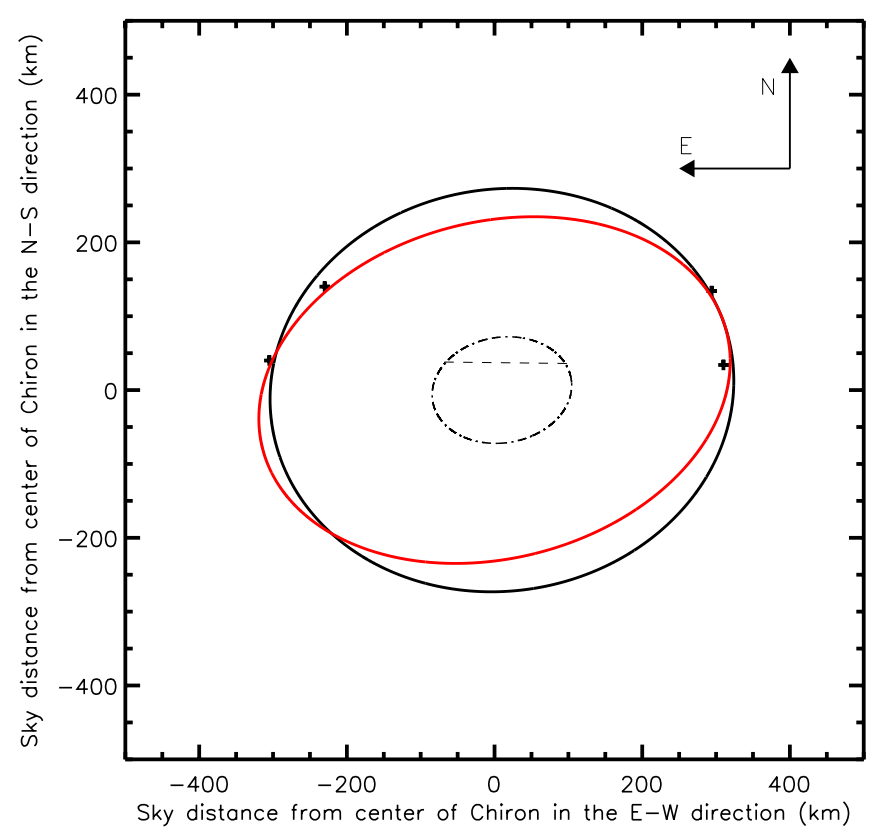

Fig. 1. Stellar occultation in 2011: the plus symbols indicate the locations in the plane of the sky where the sharp events in the lightcurves of Ruprecht (2013) took place. The upper points correspond to events detected from the Faulkes $2 \mathrm{~m}$ telescope in Haleakala, whereas the points below correspond to secondary events as seen from the IRTF $3 \mathrm{~m}$ telescope at Mauna Kea. The ellipse in thick black line shows a possible fit to the secondary events within a range of possible fits that satisfy several constraints (see text). The semi-major axis is $313 \mathrm{~km}$ and the semi-minor axis is $272 \mathrm{~km}$. The ellipse in red line is an example of another possible fit. The semi-major axis of the ellipse is $324 \mathrm{~km}$ whereas the semi-minor axis is $228 \mathrm{~km}$. The small dashed segment corresponds to the occultation chord of Chiron from IRTF. The smaller ellipse shows a tentative projected shape for Chiron that is compatible with the IRTF chord and is concentric with the ring system. The dimensions of this smaller ellipse are 190 and $143 \mathrm{~km}$.

system can be fitted to the four secondary mean events in the sky plane in Fig. 1. This is because an ellipse has five parameters but we only have four datapoints.

We can, however, add a further constraint because we know that rings are made of material in circular orbits around the main body, so the center of the body must coincide with the center of the ellipse of the rings. Also, we know that Chiron's shape cannot be spherical (because Chiron shows a double-peaked rotational lightcurve, e.g., Bus et al. 1989), so we can impose the constraint that the center of the ellipse be below the Mauna Kea chord and slightly displaced with respect to the center of the chord. This results in a set of valid solutions for a mean ellipse representing the ring system. Valid fits are obtained for ellipses with a range of position angles from approximately $11^{\circ}$ to $27^{\circ}$. The range of valid major-axis solutions is $314 \mathrm{~km}$ to $334 \mathrm{~km}$. (These values correspond to the distance of Chiron's center to the ring system, not to any of the two individual components.) As a result, $324 \mathrm{~km}$ is the average ring diameter. The valid aspect angle or tilt angle of the ring system is approximately $31^{\circ}$ to $45^{\circ}$, under the assumption that the ring system is circular, and we see it as an ellipse due to projection. Also the supplementary angles are possible, which implies that the range of angles from $135^{\circ}$ to $149^{\circ}$ is also valid. By definition, the aspect angle is the angle that the rotation axis of a body (or rings in this case) make with respect to the direction defined by the observer and the body. Using those angles we can determine approximate coordinates for the pole of the rings (which should coincide with the pole of Chiron if we assume that the rings are equatorial) with an uncertainty of around $10^{\circ}$. Two valid sets of pole direction solutions are possible because the tilt angle of the ring system has two valid sets of angles, as mentioned before. The two valid solutions have been derived with an uncertainty of $\sim 10^{\circ}$. The first solution for the direction of the pole has ecliptic coordinates $\lambda=(352 \pm 10)^{\circ}, \beta=(37 \pm 10)^{\circ}$, and the other solution has coordinates $\lambda=(144 \pm 10)^{\circ}, \beta=(24 \pm 10)^{\circ}$.

The dimensions of the smaller ellipse shown in Fig. 1 are $190 \mathrm{~km}$ and $143 \mathrm{~km}$ for the major and minor axes, respectively. This is a tentative ellipse that is compatible with the IRTF chord and is a reasonable shape for Chiron's limb. The uncertainties in those values are higher than the typical uncertainties obtained from stellar occultations with high time resolution and high time accuracy. The reason is that only one chord on the main body was obtained, and as stated above, the center of the ellipse constraint is not very tight, so families of ellipses are compatible with the sharp secondary events. The equivalent diameter of the $190 \times 143 \mathrm{~km}$ ellipse is somewhat smaller than the $218 \pm 20 \mathrm{~km}$ equivalent diameter determined from Herschel Space Observatory measurements (Fornasier et al. 2013), but thermal emission of the rings and dust around Chiron might have contributed and was not separately accounted for in Fornasier et al. (2013). Besides this, there are other equivalent diameter estimations from thermal modeling in the literature that indicate considerably smaller diameters than the $218 \mathrm{~km}$ for Chiron. We have compiled different values from the literature: Fernández et al. (2002) determined a diameter of $148 \pm 8 \mathrm{~km}$, Groussin et al. (2004) found $142 \pm 10 \mathrm{~km}$, Lebofsky et al. (1984) found $180 \pm 40 / 50 \mathrm{~km}$, and Altenhoff \& Stumpff (1995) found $198 \pm 20 \mathrm{~km}$. On the other hand, Campins et al. (1994) found values of $148 \pm 22 \mathrm{~km}$ in 1991, $176 \pm 20$ and $208 \pm 20 \mathrm{~km}$ in 1993 , and $188 \pm 12$ and $182 \pm 26 \mathrm{~km}$ in 1994. All these results are lower than the Herschel value of $218 \mathrm{~km}$, and the scatter may indicate that Chiron was observed at different rotational phases (and that Chiron could be a rather elongated body), or maybe the rings contributions or the contributions from dust could be playing a role. But differences in the thermal modeling by different authors and even mistakes or problems with the observations might also be playing a role by leading to a considerable scatter.

We must note that the IRTF lightcurve shows another brightness dip at around 8.262 UT with a similar depth to one of the secondary symmetrical events of the rings. This event therefore appears to be real, not noise. If it is real, it might be due to a small satellite or due to dust or even to an incomplete ring (an arc) because it does not have a symmetrical counterpart after the occultation of the main body. It is also curious that the number of dimming events in the IRTF and Faulkes lightcurves is apparently larger than the number of brightening events. In a pure noise distribution we should expect the number of dimming events to be nearly identical to the number of brightening events of the same magnitude, but this does not seem to be the case in these observations. This might be a hint of the existence of plenty of debris in different orbits around Chiron. However, one should be cautious because large seeing changes can sometimes produce dimmings in synthetic aperture photometry.

\subsection{Stellar occultations in 1994 and 1993}

The next step is to compare the two proposed ring systems (the two orientations) to previous occultation data in order to see if they are compatible with the observations. Although the 1994 data are poorer in quality than in 2011 and some details cannot be completely determined, it makes sense to try to check whether 


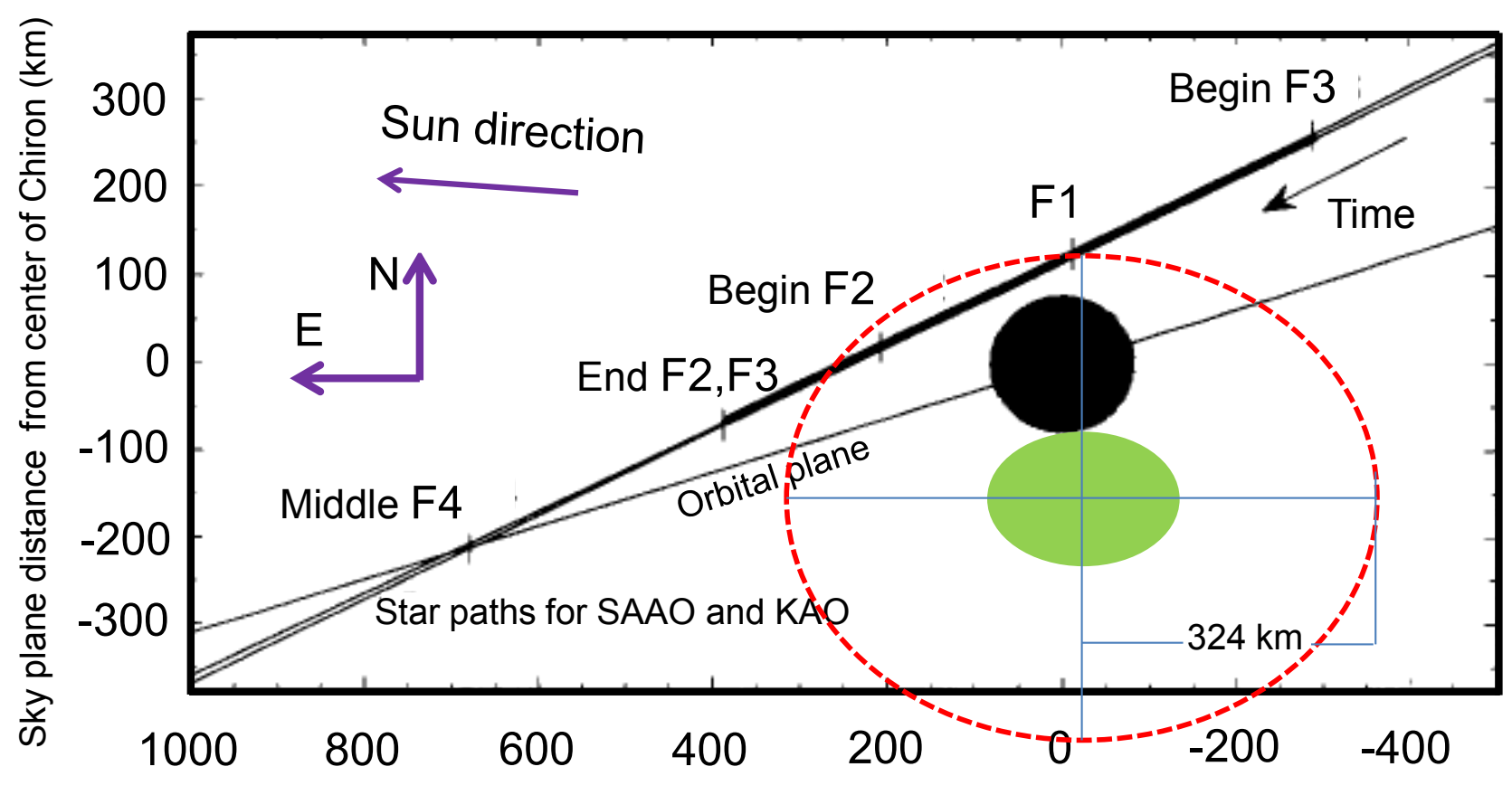

Sky plane distance from astrometric center of Chiron $(\mathrm{km})$

Fig. 2. Stellar occultation in 1994: adapted Fig. 2 of Elliot et al. (1995) showing the plane of the sky centered on Chiron (whose center was determined astrometrically, not from the occultation). The location of the relevant lightcurve features are indicated by labels F1, F2, F3, and F4. See Elliot et al. (1995) for the description of the features and to get relevant data regarding the occultation. The incomplete black circle corresponds to the position where a spherical Chiron should have been with respect to the star paths for SAAO and KAO according to astrometric measurements. The orbit plane of Chiron is shown for reference. The red ellipse is the projected shape of a ring system whose pole was determined in 2011. The corresponding position angle of the ellipse in 1994 is 1 degree, and the aspect angle is 30 degrees or 150 degrees for the two pole solutions. The intersections of this ellipse with the star paths from the two observing sites indicate where sharp occultation events would be detectable. As can be seen, the ring would clearly cause both the F1 feature and the F2 feature (whose maximum sharpness was not at the beginning of the label F2 in this figure, but slightly later, see text). The filled ellipse corresponds to a plausible position and to the shape of Chiron.

the rings are incompatible with the 1994 observations. The two ring configurations resulting from the 2011 occultation are compatible with the sharp lightcurve feature reported in Elliot et al. (1995), with label F1 and possibly the feature with label F2. This is shown schematically in Fig. 2, which is an adapted version of Fig. 2 of Elliot et al. (1995) in which we incorporate an approximate ring position compatible with the observations and the position of the main body. In our version we need to shift the main body to the southwest compared to the Elliot et al. (1995) plot. This is not a problem because the position of the main body was not determined by Elliot et al. (1995) based on the occultation itself, but based on astrometry, which is typically accurate to the $\sim 20$ mas level in optimal situations, so a displacement of around $\sim 10$ mas to the southwest, which we require, is perfectly conceivable.

The optical depth of the F1 feature is 0.92 according Elliot et al. (1995), and the optical depths of the 2011 features are around 0.7 to 1 , according to Ruprecht (2013). These are very similar values in terms of extinction and also have very similar widths ( 5 to $9 \mathrm{~km}$ and 3 to $7 \mathrm{~km}$ respectively, according the respective authors). Feature F2 in Elliot et al. (1995) is broader than the extremely sharp F1 feature, with a width of $74 \mathrm{~km}$, but the deeper part of F2, which is three to four times smaller, can be consistent with a ring feature. Maybe this feature is broader than F1 because it takes place in a part of the rings where there are fewer particles so a drop of star's light as sharp as in F1 should not be expected. It is possible that the ring system is not homogeneous azimuthally. Elliot et al. (1995) also mention two other features labeled F3 and F4 in their occultation lightcurves, but those features are very broad and shallow, and they are hardly significant above the noise; if real, they are not associated to the ring system for which much sharper brightness drops are needed. They could be associated with dust or debris around Chiron. Elliot et al. (1995) proposed a gravitationally bound coma to explain these very broad features.

Concerning the 1993 occultation, the ring configuration can be compatible with the sharp feature labeled A4 in Fig. 3 in Bus et al. (1996). In fact, the A4 feature is consistent with the F2 feature of Elliot et al. (1995). This A4 feature is $<24 \mathrm{~km}$ in width, according to Bus et al. (1996). One must also note that another ring extinction feature should have been detected in site 4 , but the feature, which should have been analogous to the F1 feature in Elliot et al. (1995), was perhaps not detected because of the longer integration time (1s) used at site 4 , in comparison to the observations in Elliot et al. (1995). The very sharp and very brief F1 would therefore not be detected with the much poorer signalto-noise ratio and poorer time resolution of Bus et al. (1996). The star that was occulted in 1994 was brighter than $m_{V}=11.9$ mag, whereas the star occulted in the 1993 event was around two magnitudes fainter.

In summary, detecting sharp features in 1993 would have been difficult. An alternative explanation for the lack of a clear detection of a counterpart feature of F1 could be that the ring system is not homogeneous and can have gaps or regions with fewer particles (which rotate with the orbital period of the particles in the rings). If the ring system were homogeneous, other lightcurve features associated with the rings should have been recorded from sites 1,2 , and 3 (Fig. 3), but we must note that 


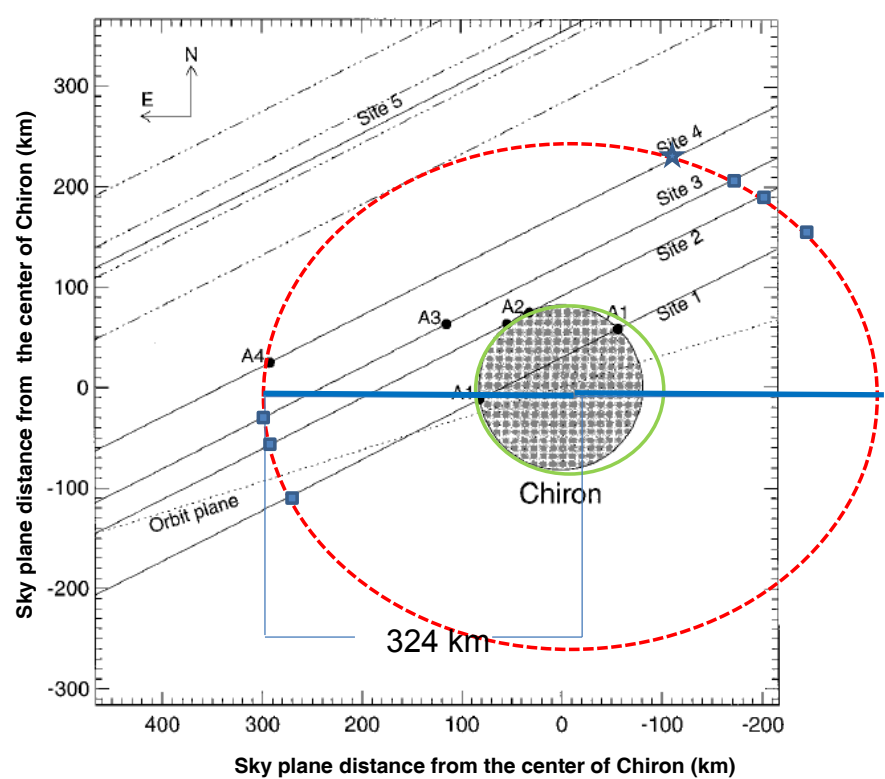

Fig. 3. Stellar occultation in 1993: Adapted from Fig. 5 of Bus et al. (1996) in which the sky plane for the region surrounding Chiron is shown. The ellipse (dashed line) shows the ring of Chiron that would cause the A4 feature and is compatible with the orientation derived from the two pole solutions derived from the 2011 occultation. The position angle is 1 degree, and the aspect angle is 30 degrees or 150 degrees. The star symbol indicates another intersection of the ring with the star path for site 4 , where another secondary event should have been detected from site 4 (see text), and the small squares show locations where secondary events should have been detected if the observations at the particular observing sites obtained data with high signal-to-noise ratio, which was not the case. The disk of Chiron is shown based on a circular fit to the occultation chords, but in green we show an alternative disk of Chiron. See main text.

the telescopes employed at those sites were all smaller than the telescope used for the detection of the A4 feature, so that the poor signal-to-noise ratio and the longer integration times used in those sites would have prevented the detection of the ring features.

This also happened in the occultation by the rings of Chariklo, where several stations used integrations that were too long and telescopes that were too small to clearly detect the ring occultations (Braga-Ribas et al. 2014). Feature A3 in Bus et al. (1996) is dubious because the brightness drop does not seem to be below the 1-sigma noise level and does not seem real at all compared with the stronger A4 feature. But even if A3 were real, it might be related to dust or debris around Chiron, not to the ring system. In Fig. 3 we show an alternative disk for Chiron to the circular fit proposed in Bus et al. (1996). The shape of Chiron is known to be non-spherical, so the projection should not be a circle. The shown elliptical limb for Chiron is concentric with the ring's ellipse. The proposed ellipse is just for illustration. It is not a real fit to the chords, but it illustrates that non-spherical shape solutions are indeed possible for explaining the observed chords. Because Chiron is smaller than a dwarf planet, its shape is very likely to be somewhat irregular, so slight deviations from a pure ellipsoid are also expected. As a result, perfect fits to the chords with no residuals using a pure ellipse are unlikely.

Combining the information from both occultations in 1993 and 1994, we should point out that for a feature at approximately the same distance from the center in both occultations, statistical likelihood is higher for a fixed ring system than a jet. Our view is therefore that at least some of the extinction features observed in 1993, 1994, and 2011 are compatible with the ring system and not compatible with a jet. We should keep in mind that Chiron was not reported to be active in those years, since no outbursts or coma was reported, so it is difficult to envision how a jet could have formed. Also, the dust size distribution derived in the 1994 occultation by Elliot et al. (1995) is very different from the typical size distribution in cometary jets observed from the ground and, in particular, to the size distribution of comet Halley observed by the Giotto spacecraft (Lamy et al. 1987). On the other hand, the very narrow ejection angle needed for the jet has never been observed in comets. These and other points raised in section 6 allow us to reject the jet interpretation for the narrow and dense extinction features. A moderate range of possible position angles and aspect angles of the ring system in the 1993 and 1994 occultations are compatible with the constraints from the ring size and from the requirement that the rings be concentric with the main body. In theory, that range of angles can be used to refine the direction of the pole derived from the 2011 occultation. However, no improvement has been obtained.

\section{Constraints from published spectra}

The spectra of Chiron reported in the literature show variable water-ice spectral features at $2.02 \mu \mathrm{m}$ and $1.5 \mu \mathrm{m}$ (Foster et al. 1999; Luu et al. 2000; Romon-Martin et al. 2003). The water ice feature even disappeared in spectra taken in 2001, as reported by Romon-Martin et al. (2003) using spectra from the $8 \mathrm{~m}$ VLT, whereas the water ice feature could be detected only a few years earlier (in 1997) by Foster et al. (1999) despite using a smaller telescope. We can explain the water-ice spectral feature and even its disappearance with a ring system.

Because we know that the rings of Saturn show prominent spectroscopic water ice features, we think that the water ice in Chiron is in the proposed rings, not on Chiron's surface. Under the assumption that the water ice is only in the rings, the mechanism that can explain the disappearance of the water ice feature is the change in the aspect angle of the rings. This aspect angle is very close to $90^{\circ}$ in 2001 (see Fig. 4) if we use the first orientation of the ring system $\left(\lambda=(352 \pm 10)^{\circ}, \beta=(37 \pm 10)^{\circ}\right)$, and around $70^{\circ}$ for the second ring pole orientation $\left(\lambda=(144 \pm 10)^{\circ}\right.$, $\left.\beta=(24 \pm 10)^{\circ}\right)$. For both ring orientations, the large decrease in the cross section of the rings can explain the disappearance of the water ice feature in 2001. The changing behavior of the water-ice absorption features in Chariklo has been recently shown to be a result of the changing aspect angle of Chariklo's rings (Duffard et al. 2014a). Thus, in Chiron we would be seeing the same effect.

According to Groussin et al. (2004), the composition that best matches both the observed reflectance spectra and albedo of Chiron corresponds to an aereal mixture of $30 \%$ of water ice plus $70 \%$ of refractory material and a common grain size of $10 \mu \mathrm{m}$. In other words, $30 \%$ of the area of Chiron would be covered with ice, and $70 \%$ of the area would be covered with dark material. Because the geometric albedo determined by Groussin et al. (2004) was 0.11 and because the typical albedo of the dark material $\left(p_{\mathrm{d}}\right)$ in outer solar system bodies is around 0.05 to 0.08 , the albedo of the water ice particles $\left(p_{\mathrm{w}}\right)$ can be obtained from $0.11=0.7 p_{\mathrm{d}}+0.3 p_{\mathrm{w}}$, hence $p_{\mathrm{w}}=0.27$ if we use $p_{\mathrm{d}}=0.05$ and $p_{\mathrm{w}}=0.19$ if we use $p_{\mathrm{d}}=0.08$. If we assume that the water ice particles are all in Chiron's rings, the work by Groussin et al. (2004) implies that the rings must be at least $30 \%$ of the total area of Chiron's system. But we think that the area of the rings is even slightly larger than $30 \%$ of the total area. The area of Chiron's main body is $\pi r^{2}$ with $r=90 \mathrm{~km}$ (an intermediate 

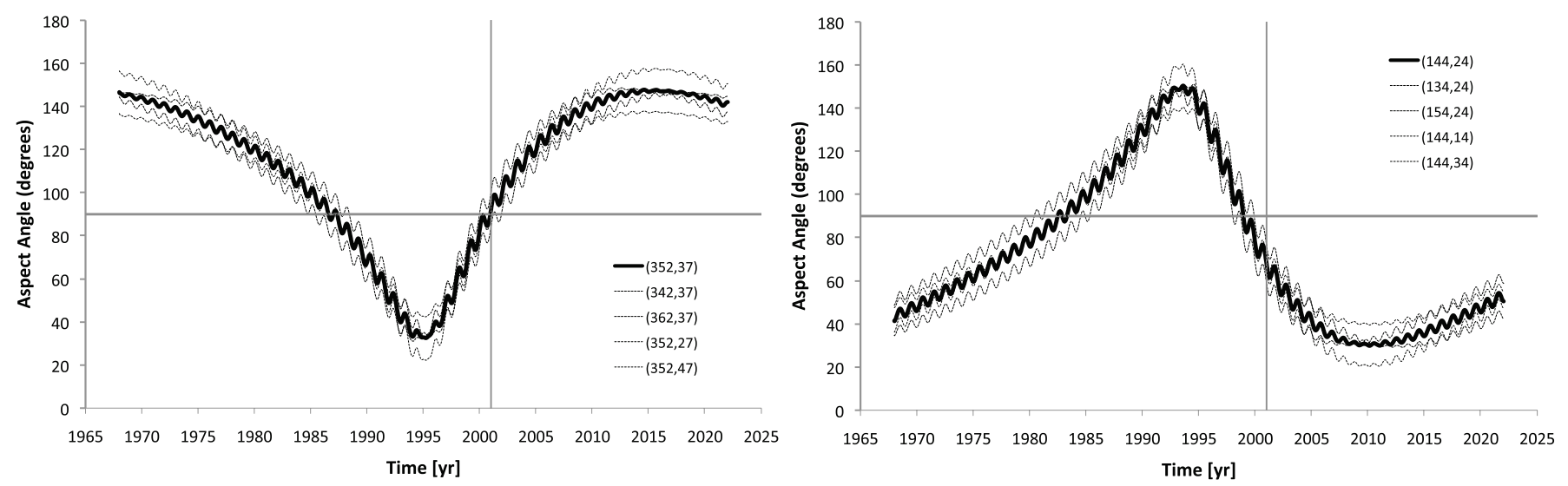

Fig. 4. Aspect angle versus time. Left panel: the continuous line shows the aspect angle of Chiron's rings as a function of time for the nominal pole direction that we obtained from the analysis of the occultations of the rings $\left(\lambda=352 \pm 10^{\circ}, \beta=37 \mathrm{deg} \pm 10^{\circ}\right)$. The rest of the lines correspond to solutions within the uncertainties of the nominal pole. The vertical and horizontal lines are shown to highlight that in 2001 the aspect angle of the rings was 90 degrees. The right panel shows the aspect angle as a function of time for the other pole direction $\left(\lambda=(144 \pm 10)^{\circ}, \beta=(24 \pm 10)^{\circ}\right)$.

size of all the values mentioned in the previous section), whereas that of the rings is approximately $2 \pi r_{\text {ring }} w_{\mathrm{p}}$ where $r_{\text {ring }}$ is the radius of the rings $(324 \mathrm{~km})$, and $w_{\mathrm{p}}$ is the combined width of the two rings seen from the observer $(10 \mathrm{~km}$, from the 2011 occultation). This means that the area of the rings can be around $50 \%$ of the total Chiron system. With the new percentages, the albedo of Chiron's system is $0.11=0.5 \times 0.05+0.5 \times p_{\mathrm{w}}$ so we can derive the albedo of the ring particles $p_{\mathrm{w}}$. The new value is $p_{\mathrm{w}}=0.17$ if we use $p_{\mathrm{d}}=0.05$ and $p_{\mathrm{w}}=0.14$ if we use $p_{\mathrm{d}}=0.08$. Thus we think that the rings geometric albedo is in the 0.14 to 0.17 range based on the spectroscopy constraints, but could be up to 0.27 if the rings fractional area is around $30 \%$ of the total system. This range of albedo values will be used in the next section to model Chiron's historical brightness evolution.

Because the 2001 spectra were taken while Chiron was experiencing a clear activity outburst, it might be argued that the dust released in the outburst would dominate the reflected light and thus this could hide the water ice spectral feature. However, we have simulated this effect using the measured brightness increase of Chiron in 2001 and assuming that all this increase went to spectrally featureless dust. This means changing the areal mix in the spectroscopy models to around $85 \%$ of spectrally featureless material. Even though the water ice spectral feature gets somewhat diluted in the simulations with $85 \%$ of spectrally neutral dust (see Fig. 8 of Groussin et al. 2004), the water ice band is still detectable, so the outburst cannot completely hide the water ice feature. Another mechanism must therefore be in action or combined to the outburst to hide the water-ice spectral feature. Maybe an instrumental problem is the explanation, but the change in the aspect angle of the rings mentioned in the first paragraph of this section is the easiest explanation.

\section{Constraints from long-term photometry}

Using a compilation of absolute magnitude measurements of Chiron at different epochs from Belskaya et al. (2010), we realized that the two deep maxima in absolute magnitude (brightness minima) coincide with the epochs when the proposed rings were edge-on with respect to an Earth observer (aspect angle of 90 degrees). In Fig. 5 we plot the absolute magnitude and the sine of the aspect angle of the rings for the two pole solutions, as a function of time. We do this to illustrate when the rings are edge-on (maxima of the curves) and to compare the maxima in the curves with the absolute magnitude measurements. As can be

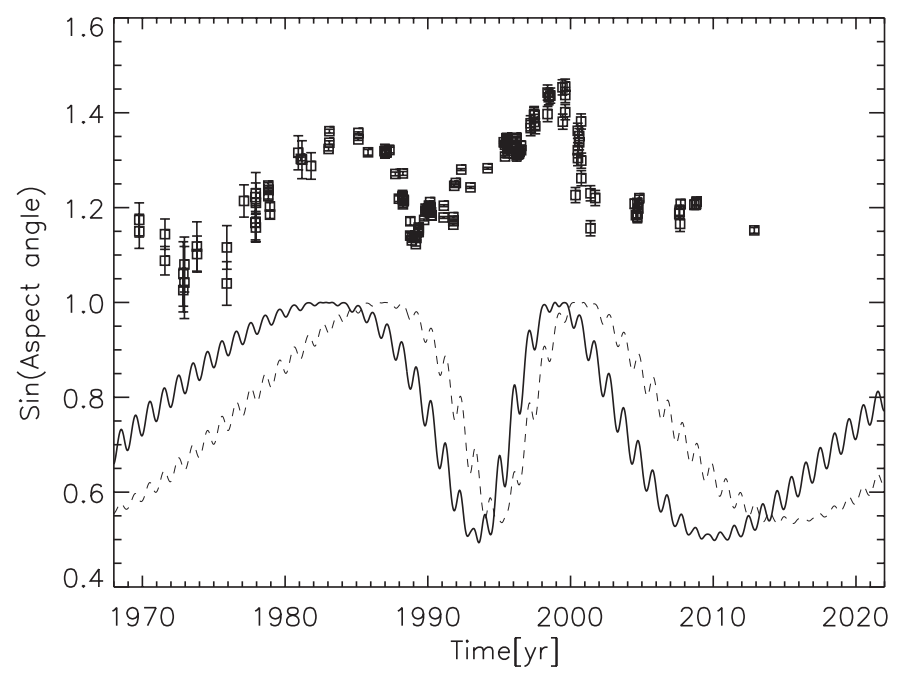

Fig. 5. Coincidence of the brightness minima with aspect angle near 90 degrees. With a continuous line we show the sine of the aspect angle of Chiron as a function of time using the preferred pole solution $\left(\lambda=144^{\circ}, \beta=24^{\circ}\right)$. The dotted line corresponds to the other solution for the pole direction $\left(\lambda=352^{\circ}, \beta=37^{\circ}\right)$. The square symbols represent the absolute magnitude of Chiron from Belskaya et al. (2010) divided by a factor 5 for easier viewing. As can be seen, there is coincidence between aspect angle and absolute magnitude, but there is a shift between the aspect angle maxima and the absolute magnitude maxima for the $\lambda=352^{\circ}, \beta=37^{\circ}$ pole solution.

seen, the second pole solution $\left(\lambda=(144 \pm 10)^{\circ}, \beta=(24 \pm 10)^{\circ}\right)$ gives a better match to the times when the maxima in absolute magnitude (brightness minima) are reached. Our explanation of this coincidence is that the rings have an important effect on the long-term brightness of Chiron's system, and we built a simple model for the brightness of Chiron's main body and its ring system, in which a large portion of the brightness comes from the rings. A similar model was built to successfully explain Chariklo's absolute magnitude changes over time (Duffard et al. (2014a).

With the second pole direction $\left(\lambda=(144 \pm 10)^{\circ}, \beta=(24 \pm\right.$ $\left.10)^{\circ}\right)$, an overall match to the absolute magnitude measurements of Chiron (except the parts with large brightness excursions due to activity outbursts) can be obtained. Activity outbursts were 
observed in 1989 (e.g., Meech \& Belton 1989) and in 2001 (Romon-Martin et al. 2003). Also, Bus et al. (2001) suggest that an outburst could have taken place around the 1970s. The key feature of the model is the inclusion of the changing aspect of Chiron's rings.

The model is very simple and takes the total flux coming from both Chiron's nucleus and its rings into account:

$$
\frac{F_{\text {tot }}}{F_{\text {Sun }}}=A_{\mathrm{p}} p_{V} f(\alpha)+p_{V}^{\text {Ring } 1} f^{\prime}(\alpha) 2 \pi W_{1} a_{1} \mu+p_{V}^{\text {Ring } 2} f^{\prime}(\alpha) 2 \pi W_{2} a_{2} \mu
$$

where $F_{\text {tot }}$ is the flux received at Earth, $F_{\text {Sun }}$ the solar flux at Chiron, $A_{\mathrm{P}}$ the projected area of Chiron's nucleus, $p_{V}$ the geometric albedo of the main body, $f(\alpha)$ the solar phase function, $p_{V}^{\text {Ring1 }}$ the albedo of the first ring, $f^{\prime}(\alpha)$ the solar phase function of the ring, $\mu$ the absolute value of the cosine of the observer zenith angle from the rings (which coincides with the cosine of the rings' aspect angle), and $2 \pi W_{i} a_{i} \mu$ is the projected area of the rings, where $W_{i}$ is the radial width of the $i$ ring, and $a_{i}$ the radial distance of each ring to the main body.

The solar phase function $f(\alpha)$ we take as equal to 1 for all phase angles, which is a valid approximation because we know that the phase angle dependence is weak (around a few percentage points of magnitude per degree according to Belskaya et al. 2010), and the phase angle ( $\alpha$ ) in Centaurs is only a few degrees at most. We assume the same for the rings' phase function.

We take the albedo of the rings as 0.17 from the spectroscopy constraints, and $a_{i}$ was taken as $324 \mathrm{~km}$ from the occultation results. The geometric albedo of Chiron's main body, $p_{V}$, was taken as 0.08 from Lebofsky et al. (1984) because the observations in that work were performed when the ring was almost edge on, so the albedo determination was not contaminated by the rings. Besides, the surfaces of the centaurs are thought to be like those of the comets, whose geometric albedo is typically around a few percentage points. Comets and Centaurs are dynamically linked, so it seems reasonable that both populations have similar albedos. It is important to note that $\mu$ for the different epochs depends on the rings' pole direction. Also, the projected area $A_{\mathrm{p}}$ was computed by assuming that Chiron is a triaxial ellipsoid with the following dimensions: $a=100 \mathrm{~km}, b=84 \mathrm{~km}$, and $c=70 \mathrm{~km}$, where $a, b, c$ are the semimajor axes of the triaxial ellipsoid describing the body.

These dimensions are obtained by using the $a / b$ axial ratio determined for Chiron by Groussin et al. (2004) and putting the constraint that the equivalent diameter of a sphere is $\sim 180 \mathrm{~km}$. We use $180 \mathrm{~km}$ because this is an average value among all the values reported in the literature, as already mentioned in previous sections. The axial ratios were also used in the subsequent section to explain the amplitude of the rotational lightcurves. The model does not take the area of the rings occulted by the main body into account, but this is a tiny correction and would only affect a narrow range of aspect angles.

In Fig. 6 we show the brightness of Chiron and its rings from the model, together with the measurements of absolute magnitude of Chiron at different epochs compiled by Belskaya et al. (2010). These data are mostly comprised of the data by Duffard et al. (2002) and Bus et al. (2001)

In this model there are no free parameters because all the parameters are obtained from either the literature or from our results in the previous sections. However, given the uncertainty range in some of these parameters, it is useful to test the effect of some of them. The parameter that has the largest relative uncertainty is the geometric albedo of the rings, so we tested its

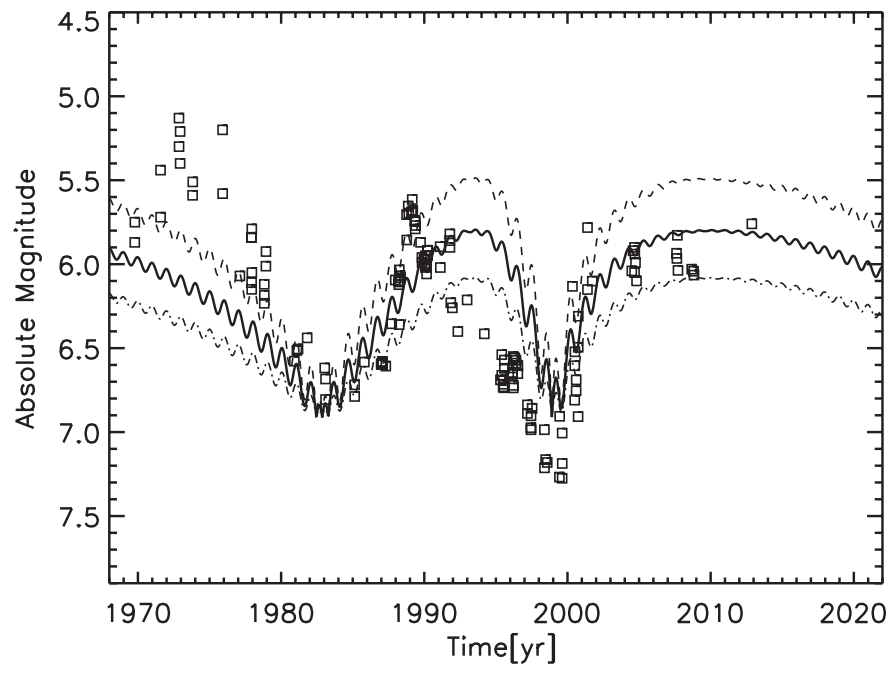

Fig. 6. Model to explain the absolute magnitude in $V$ band. The continuous line shows the results from the nominal model that can explain the overall photometry of Chiron from Belskaya et al. (2010, square symbols). The upper and lower dashed lines represent models with ring albedos of 0.1 and 0.27 , respectively.

effect by using a 0.1 albedo and a 0.27 albedo in addition to the nominal 0.17 value derived from the spectroscopy.

The lower line in Fig. 6 corresponds to a model with a geometric albedo of 0.1 for the rings. The central line shows the model with the nominal parameters (geometric-albedo of the rings 0.17 ), which provides better agreement with the observations. In the upper line we present a model with a geometric albedo of 0.27 for the rings. It is important to note that the contribution from the rings to the total visible flux is similar to that of the projected area of Chiron (except when the rings are seen nearly edge-on, at aspect angles around 90 degrees, when the rings do not contribute). This can explain a greater-than-one magnitude difference from maxima to minima. Chiron's exact shape is not a relevant factor for explaining the overall absolute magnitude measurements. A ring system is the only way that we can think to cause a large magnitude change of around one or more magnitudes. As already stated, the model fails to reproduce the brightness local maxima that are related to activity outbursts. We know that Chiron has been an active body, and outburst episodes are unpredictable. Besides that limitation, the model does not completely reproduce the absolute magnitude of the observed brightness minima because the observations show that the two brightness minima have different values. We can explain a deeper brightness minimum in 2000 than in 1983 if the albedo of Chiron had decreased significantly in the 20 years from 1983 to 2000, which would be difficult to explain (unless there is some dark dust recoating the nucleus or extinguishing its signal). The same would be true if the size of Chiron had changed considerably in $\sim 20$ years, which is even more difficult to justify from a physical point of view. But there is an alternative way to explain the difference in the two minima, and we can model it easily. If we add the contribution of a cloud of debris or dust whose brightness decays exponentially with time, with a time constant of around ten years (see Fig. 7), we can explain the different depths of the two observed brightness minima. The only other parameters in the model are the albedo of the coma particles, which we set to 0.06 (a typical value of comet dust albedo), and the initial cross section of the cloud of particles, which we determined iteratively to be $\sim 85000 \mathrm{~km}^{2}$. This area 


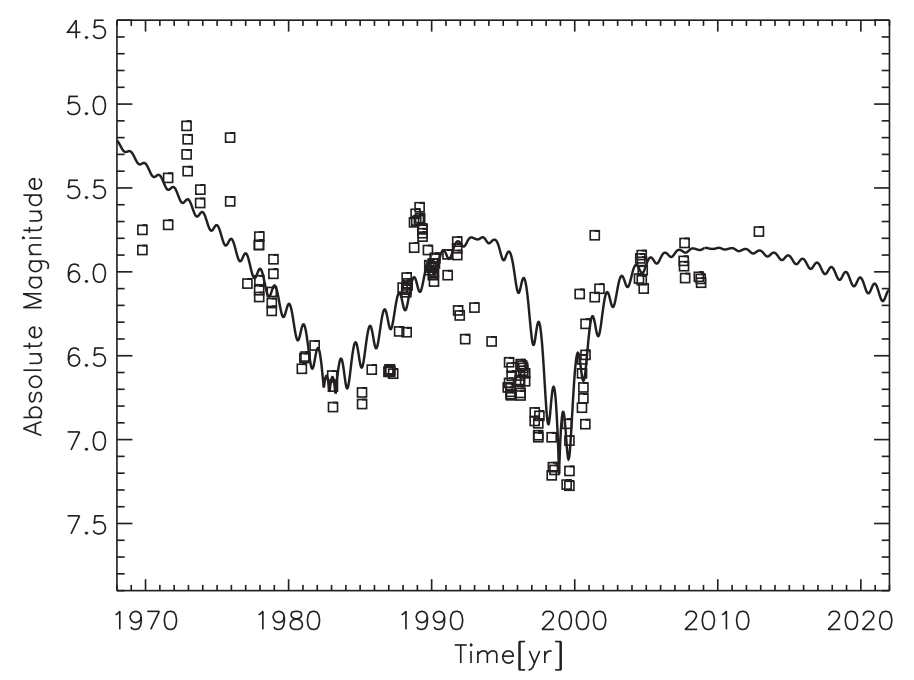

Fig. 7. Model that includes a vanishing coma to explain the absolute magnitude in $V$ band. In continuous line we show the results from the model discussed in the text but including the contribution of an exponentially decaying coma. The observations as compiled in Belskaya et al. (2010) are shown as square symbols.

is comparable to the total cross section of Chiron and its rings. Mathematically, the modified model can be expressed with the following equation:

$$
\begin{aligned}
\frac{F_{\text {tot }}}{F_{\text {Sun }}}= & A_{\mathrm{p}} p_{V} f(\alpha)+p_{V}^{\text {Ring1 }} f^{\prime}(\alpha) 2 \pi W_{1} a_{1} \mu \\
& +p_{V}^{\text {Ring2 }} f^{\prime}(\alpha) 2 \pi W_{2} a_{2} \mu+A_{\mathrm{c}} p_{\mathrm{d}} \exp \left(-\left(t-t_{0}\right) / \tau_{\mathrm{d}}\right)
\end{aligned}
$$

where $A_{\mathrm{c}}$ is the initial scattering cross section of the cloud, $p_{\mathrm{d}}$ the geometric albedo of the dust, $\tau_{\mathrm{d}}$ the decay time, $t$ time, and $t_{0}$ is the initial time at which the dust cloud starts to decay.

Because there are hints of plenty of debris or dust around Chiron, it seems plausible that some debris may be escaping from Chiron with an exponential behavior, and therefore the cloud brightness could decay exponentially. Elliot et al. (1995) proposed that Chiron is surrounded by a bound coma to explain some of their observations. It seems possible that such a bound coma or cloud is not completely bound on time scales of tens of years and that some material is escaping slowly. An exponentially decaying coma has already been proposed for Chiron by Meech \& Belton (1990) and Duffard et al. (2002), although with different time constants. Also, it is important to note that the absolute maximum in the photometry around 1973 is best explained in this scenario. In this modified model, we set the start of the decay $\left(t_{0}\right)$ at the first year for which we have data (1970), but the decay might have started in 1973. This would provide an even better agreement with the photometry, but at the expense of adding more free parameters to the model, such as the time of outburst and the cloud growth parameters, for which we do not really have good constraints. Many other sophistications are possible for the model, including resurfacing or coating mechanisms, and modeling the outbursts, but they are not straightforward to implement. Here we just presented some rough ideas. In summary, the model whose key feature is a ring system with changing cross section is consistent with the overall behavior of Chiron's brightness.

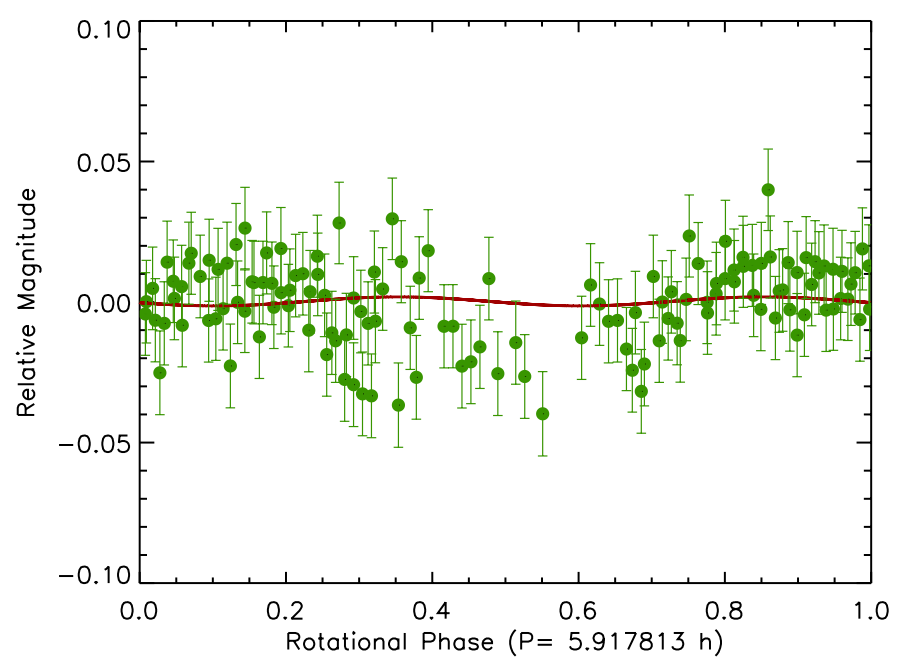

Fig. 8. Lightcurve of Chiron. Rotational lightcurve obtained from the CAHA 1.2 m telescope in 2013. A two-term fit (two oscillations per rotation cycle) is shown on top of the data. The Julian date for zero phase angle is 2451783.41511 .

\section{Lightcurve amplitude}

We got $250 \mathrm{~s} R$-band exposures of Chiron with the $4 \mathrm{k} \times 4 \mathrm{k}$ DLR-MKIII CCD camera of the $1.23 \mathrm{~m}$ Calar Alto Observatory telescope in Almeria, Spain. The field of view of the instrument was $21.5 \times 21.5$ arcmin, and the plate scale was 0.63 arcsec per pixel. The images were obtained on October 9 and 10, 2013. The telescope was tracked at the sidereal rate. Bias frames and twilight flatfield frames were taken each night to calibrate the exposures. No sign of coma was observed in the images. The profiles of Chiron were entirely identical to stellar profiles, so no signs of activity were detected. From the images we obtained time series relative photometry using the same techniques described in Thirouin et al. (2012). We used several synthetic aperture radii and chose the results that gave the lowest scatter. The final time series relative photometry is given in Table 1. From the photometry we derived the rotational lightcurve by phasing the photometry to the known $5.917813 \mathrm{~h}$ period of Chiron (Bus et al. 1989; Marcialis \& Buratti 1993). We fitted a two-term series to the lightcurve, because we know that the lightcurve is double-peaked (Bus et al. 1989; Marcialis \& Buratti 1993). The derived lightcurve amplitude turned out to be $0.003 \mathrm{mag} \pm 0.015$, so much smaller than the 0.088 mag amplitude derived by Bus et al. (1989) in 1986 and 1988. The lightcurve and the fit are shown in Fig. 8.

We also derived time series photometry of Chiron from CCD images that were obtained at the CASLEO $2.15 \mathrm{~m}$ telescope on August 26 and 28, 2000, but had not been analyzed. The images were obtained with a TK $1 \mathrm{~K}$ CCD camera in the $R$-filter and consisted of $60 \mathrm{~s}$ to $100 \mathrm{~s}$ exposures. The field of view of the instrument was $4.6 \times 4.6$ arcmin, and the plate scale was 0.54 arcsec per pixel. The telescope was tracked at sidereal rate. Bias frames were available, but flatfield frames were not, so we applied no flatfield correction. This resulted in slightly poorer photometry than what would have been possible, but the results are still useful. We derived the time series photometry by using the same techniques as for the $1.23 \mathrm{~m}$ telescope images. The results are given in Table 1 , and the rotational lightcurve is presented in Fig. 9. In this case the observations did not cover a full rotation of Chiron, but were enough to cover a substantial part of it and allowed us to fit an amplitude of $0.097 \pm 0.039$ mag. 


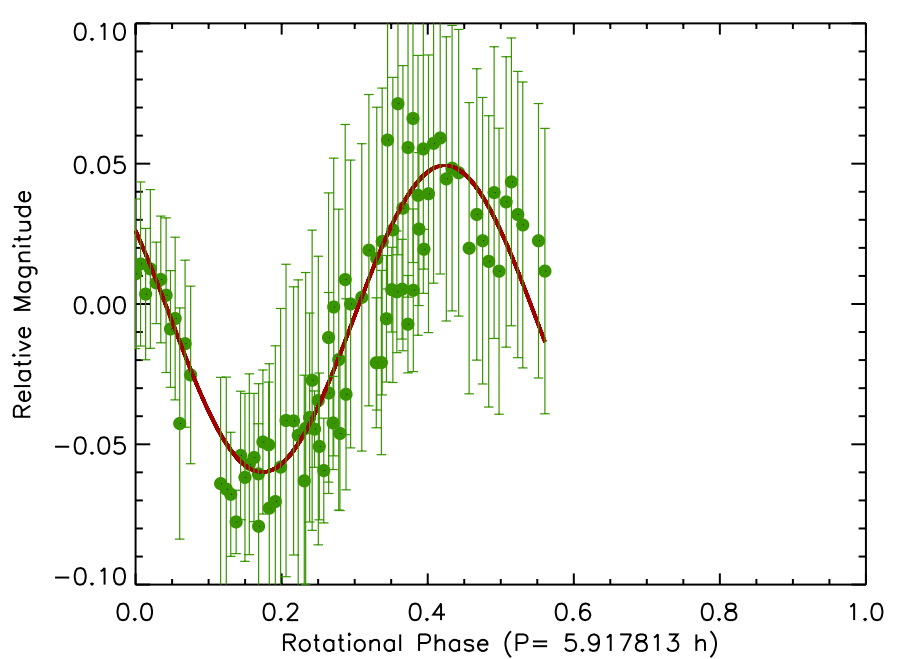

Fig. 9. Lightcurve of Chiron. Rotational lightcurve observed from CASLEO $2.15 \mathrm{~m}$ telescope in 2000. A two-term fit (two oscillations per rotation cycle) is shown on top of the data. The Julian date for zero phase angle is 2451783.41511 .

We modeled the shape of Chiron's nucleus as a triaxial ellipsoid, which is a first-order approximation. The cross section variations cause the main part of the rotational variability. For a triaxial ellipsoid, the amplitude of the lightcurve $(\Delta m)$ is, according to Binzel et al. (1989),

$\Delta m=2.5 \log \left(\frac{a}{b}\right)-1.25 \log \left(\frac{a^{2} \cos ^{2} \xi+c^{2} \sin ^{2} \xi}{b^{2} \cos ^{2} \xi+c^{2} \sin ^{2} \xi}\right)$

where $\xi$ is the aspect angle, and $a, b$, and $c$ are the semi-axes lengths of the ellipsoid with $a>b>c$. This kind of model has already been used to determine the pole of Pholus, another centaur Tegler et al. (2005). The aspect angle is related to the ecliptic longitude and latitude of the spin vector $\left(\lambda_{\mathrm{p}}, \beta_{\mathrm{p}}\right)$ by standard equations (e.g., Tegler et al. 2005).

However, this simple model, which is commonly used to model asteroid's lightcurve amplitudes, does not take the brightness of rings into account. As pointed out before, this contribution is significant, and it attenuates the amplitude of the lightcurve whenever the rings are not edge-on. We computed the decrease in the amplitude as a function of time by adding the ring flux contribution as in Eq. (1).

Using axial ratios $a / c$ of 1.43 and $b / c$ of 1.2 and the preferred pole direction $\lambda_{\mathrm{p}}=144^{\circ}, \beta_{\mathrm{p}}=24^{\circ}$ derived from the occultation, we can explain the lightcurve amplitude of the Bus et al. (1989) data (which corresponds to data obtained in 1986 and 1988), the amplitude in subsequent years compiled by Groussin et al. (2004) and our new results obtained in the present work. All this is depicted in Fig. 10. The value of the $a / c$ axial ratio is fairly irrelevant, because the lightcurve amplitude basically depends on the $a / b$ ratio alone. We chose a ratio $a / c$ rather arbitrarily, although it is consistent with the occultation of the main body. Other values of $a / c$ can also result in good fits provided that the axial ratio $b / c$ is chosen so that $a / b$ is around 1.16 . This $a / b$ axial ratio of $1.16 \pm 0.03$ was found by Groussin et al. (2004). It is also important to note that some degree of rotational variability can be due to albedo variegations on Chiron's surface. The model is a first-order approximation. Nevertheless, the agreement of the model with the observations is remarkable. The reduced $\chi^{2}$ of the fit is 1.9 . The pole solution $\lambda_{\mathrm{p}}=352^{\circ}, \beta_{\mathrm{p}}=37^{\circ}$ does not produce a satisfactory fit to the observed amplitudes.

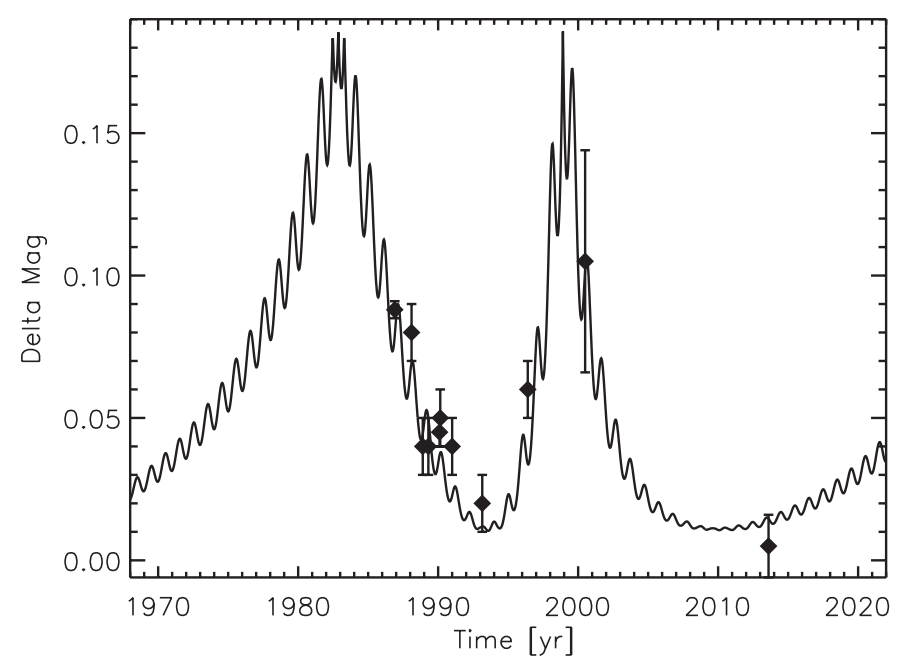

Fig. 10. Amplitude versus time: in continuous line we show the amplitude of the rotational lightcurve for an assumed triaxial shape of Chiron (with axial ratios $a / c=1.43$ and $b / c=1.2$ ) and using the nominal pole direction determined from the stellar occultations $\left(\lambda \sim 144^{\circ}, \beta \sim 24^{\circ}\right)$. The diamond symbols represent the observed amplitudes for the specific dates of observation (see text). As can be seen, the model provides agreement with the observations.

\section{Other support for the existence of a ring system}

According to Ruprecht (2013), the very sharp occultation features in 2011 were symmetric jet-like features that could be caused by a near-circular arc or shell of material ejected by Chiron and expanding away from the nuclear surface produced by one or more active regions on the nuclear surface. But a shell would cause an occultation along the whole chord from the start to the end of the shell, not sharp isolated features. One can argue that the sharp features could be caused by the increased apparent optical depth of a narrow spherical shell at nearly tangent angles from the observer, but at least some degree of occultation should be detectable along the chords, but this was not the case. Besides this, the shell would have to be ellipsoidal, not spherical, to explain the features detected from the two observing sites, when they are projected on the sky plane.

To our knowledge, no ellipsoidal shells have ever been observed in a comet. And to make matters worse, there should be two concentric narrow shells with a small gap in between. Such narrow shells have never been observed in cometary coma. As a result, the shell explanation has many problems. Regarding jets, we cannot explain why they would give symmetrical configurations, and again, they should give rise to a double structure with a small gap in between. Also, it must be pointed out that Chiron was in a quiet state at the epochs of the occultations. It suffered no outburst and did not show coma in 1993, 1994, and 2011. Apparently there was thus no source from which a jet or a shell could emanate. In addition to all this, a jet or a shell cannot explain the sharp minima in Chiron's long-term brightness. Therefore, not only are the rings a good explanation for all the features mentioned in the previous section, but alternative scenarios, such as a shell or a jet, also fail to explain the observed phenomena.

The presence of rings can be related to the considerable scatter in the values of the equivalent diameter and albedo of Chiron reported in the literature from thermal observations, because the ring contribution could be important, at least in the optical, and it strong depends on the aspect angle. It is also remarkable that the 
geometric albedo of the rings determined from the spectroscopic constraints is consistent with the geometric albedo of the rings that is used in the photometry model to explain the long-term brightness behavior of Chiron. On the other hand, the widths of the extinction features, their separations, and their optical depths are, as already mentioned in the introduction, nearly identical for Chiron and for Chariklo. These are clear indications that Chiron's extinction features are probably caused by the same type of structure as in Chariklo. Also, the radius of the rings can be a further indication: the radius of the proposed ring system in Chiron from our results is $324 \pm 10 \mathrm{~km}$, around a factor 0.82 smaller than for Chariklo, which is around $400 \mathrm{~km}$ (Braga-Ribas et al. 2014). This seems reasonable given that Chiron is smaller than Chariklo.

For plausible densities of both Chiron and a satellite, the ring system is within the Roche limit in both Chariklo and Chiron. Therefore, they would both have ring systems with similar features. Nevertheless, as already stated, Chiron's ring system would be brighter compared to Chiron than Chariklo's rings compared to Chariklo. Along with this, Chiron may have dust or debris in orbit, not just a ring system. All this might indicate that the proposed ring system could be younger than that of Chariklo, if the rings are formed from collisional or satellite disruption processes. See discussion section.

\section{Discussion}

The possbility that not only Chariklo but also Chiron, the second largest centaur after Chariklo, also has a ring system is remarkable. This might be a hint that the origin of the rings could be related to phenomenology specific to centaurs, or perhaps rings are common in the outer solar system from Jupiter and beyond. Also, as is the case for Chariklo's ring system, Chiron's ring system has a gap and the rings seem to have sharp edges. Both ring systems might thus be confined by shepherd satellites, as proposed in Braga-Ribas et al. (2014).

The rings in centaurs may come from material released from the main body after an impact, but the rings may also result from the destruction of a small satellite or from the release of material from a satellite upon impact. If the rings' origins have to do with collisions on the main body, smaller centaurs (which have a smaller cross section) would have a lower probability of developing rings. This might be tested in the future if we could observe occultations by Centaurs of different sizes. In these last two cases, the rings would result from two-step processes: first an event that generates one or several satellites and then a collision on a satellite. Whether the satellite or satellites result from the ejecta of direct collisions to the main bodies or result from other phenomena (for instance, the satellites were captured while the body dwelled in the trans-Neptunian belt or the satellites were formed from rotational fissions) needs to be investigated as well.

Several studies indicate that satellites may be very common in the trans-Neptunian belt and more common the closer they get to the primary object (Noll et al. 2008). They can come from very different formation scenarios. Even though capture is in general the favored mechanism, for small satellites close to the primaries, the main causes can be collisions or even primordial rotational fissions of the primaries. A shattering collision with a satellite in the lifetime of a centaur releasing material equivalent to a $1 \mathrm{~km}$-radius body would occur only if the current flux of impactors in the centaur region is at least one or two orders of magnitude higher than what was derived in Levison et al. (2000). Such an enhanced flux rate compared to the one in Levison et al. (2000) would be consistent with the fact that the impact of a nearly $\mathrm{km}$-sized object has been observed on Jupiter after the Shoemaker-Levy 9 impact, and this requires a larger impact flux than previously thought (Sánchez-Lavega et al. 2010). In this view, therefore, it appears that the impact rate in the outer solar system has been underestimated in the past.

The rings might also come from sublimation-driven activity events on the satellites, but this would require that the satellite or satellites have more volatile material than the primary itself. Besides this, fine-grained material, which is typical of cometary activity, would easily disperse on small time scales thanks to solar radiation and would not easily form a long-lasting ring. Therefore, sublimation-driven activity on a satellite does not seem to be a very plausible cause for the rings' material.

Thus far, several occultations of bright stars caused by large TNOs have been recorded: from historical occultations by Pluto (Pasachoff et al. 2005; Elliot et al. 2003, 2007; Person et al. 2008; Young et al. 2008) to occultations by Varuna and a few others (Sicardy et al. 2010, 2011; Ortiz et al. 2012; Braga-Ribas et al. 2013). For some of these occultations, the stars involved were bright enough that a very good signal-to-noise ratio was achieved. Sharp secondary events caused by rings should therefore have been recorded already if rings were very common in trans-Neptunian objects (TNOs). This could mean that the rings in TNOs are not common, and in this interpretation, rings of centaurs would not be formed in the trans-Neptunian belt. But the lack of detecting any ring in TNOs could also be due to size concerns, because we have recorded the occultations of only the largest TNOs. Except for the occultation of (55 636) 2002 TX $_{300}$ (Elliot et al. 2010), which is a very peculiar TNO belonging to the so-called "Haumea family" and which is not very representative of most of the TNOs (Licandro et al. 2006), there are no recorded occultation events of TNOs in the size range of Chariklo and Chiron (180 km to $260 \mathrm{~km}$ in equivalent diameter). The smallest representative TNO whose occultation has been recorded is $2002 \mathrm{KX}_{14}$ (Alvarez-Candal et al. 2014). In that work an equivalent diameter between 365 and $455 \mathrm{~km}$ is reported, but no secondary events were observed despite the 0.265 s time resolution, which would have allowed the detection of rings of similar optical depths to those reported for Chariklo. And even if (55 636) $2002 \mathrm{TX}_{300}$ is not a very representative TNO, it must be mentioned that no brief secondary events were detected by Elliot et al. (2010), despite the very high time resolution (0.072 s) and good signal-to-noise ratio (105 for a hypothetical 1s integration). Their Fig. 1 clearly shows that the best lightcurve does not have brief sharp events prior to or after the main occultation.

There is, therefore, a slight indication that rings may be formed only in the Jupiter to Neptune solar system region where centaurs reside rather than in the trans-Neptunian region. This could perhaps be due to particularly enhanced collisional rates compared to the trans-Neptunian region. In this view the rings would form from collisions on the main body or on a satellite that was either primordial or captured while the object was in such a region.

One can wonder why rings have not been identified in main belt asteroids despite there having been many occultations observed with good time resolution. The lack of rings in the main asteroid belt may be related to the average impact speed in the asteroid belt compared to the impact speeds in the regions where the centaurs reside. But this is too speculative at the current time. Outside the main asteroid belt, in the Jupiter Trojan's reservoir of small bodies, there are fewer occultations observed than in the main asteroid belt. It may be interesting to point out that Agamemnon ((911) or 1919 FD), a large Jupiter Trojan, showed a secondary and sharp event during an 
occultation (Timerson et al. 2013). This event has been interpreted as an indication of a small satellite of a few km, but perhaps it reveals the presence of a ring that was not detected in the other occultation chords simply because of insufficient signal-tonoise. The secondary event was only recorded by the observer who used the largest instrument, a $0.36 \mathrm{~m}$ telescope, whereas the other observers used $5 \mathrm{~cm}$ binocular video systems, which are too small for detecting secondary events of this sort. But a ring should have produced two symmetrical secondary events, not just one.

Therefore, the best explanation so far seems to be a satellite, although a partial or incomplete ring (an arc) should not be discarded yet. It must be noted that Agamemnon has large excursions of its magnitude as reported to the Minor Planet Center compared to the predictions from JPL ephemeris, much like Chiron's case. This large variability could be caused by a partial ring. We have done a search for Trojan asteroids that show brightness excursions in a similar to what Cikota et al. (2014) did to find main belt comet candidates. The only Trojans clearly showing large variations were Agamemnon, Achilles, and Aneas.

Concerning the historical absolute magnitude of Chiron versus time, we can explain the brightness minima and the overall behavior with the ring system, which is remarkable. So far there has been no model that could explain the changing absolute magnitude of Chiron. Of course, there are details that cannot be fit by our model, particularly at the epochs of Chiron's activity outbursts, when episodes of sudden dust release take place. Concerning the activity outbursts, one could think that there are sublimation-driven active regions within the rings or at a high latitudes on Chiron's surface that get activated when the solar incidence angle is appropriate. However, as we show in the aspect angle plots versus time, the same aspect angles as those in the outburst epochs (for example, 1988-1989 and 2001) repeated at other epochs, but there were no activity outbursts in those years. The activity therefore cannot be completely controlled by insolation factors.

From our study we can propose other scenarios that would give rise to the transient development of coma. A possible scenario could be the fallback of debris that has not been accreted to the rings or the fall of material from the rings. The impact of debris on the surface of Chiron might suddenly release dust at unpredictable times. These outbursts would not be sublimationdriven, unlike the usual comet outbursts. If the debris follows a size distribution, it may be possible that small outbursts caused by debris of small size could be more frequent than the two outbursts detected thus far and these smaller outbursts would be detectable in accurate time series photometry measurements. In summary, we are proposing that activity outbursts could be caused by the fallback of material that may be part of the ejecta of an old collision of a small body with Chiron or with a satellite. Part of the ejecta could form a disk that could evolve to a ring and part of the ejecta could eventually fall back and cause transient comae.

Finally, we would also like to speculate on another interesting topic. An intriguing possibility is whether the color bimodality observed in the color distribution of centaurs (Peixinho et al. 2003, 2012) is due to centaurs with rings and centaurs without rings. Centaurs with rings would have a bluer or a more neutral color than the other centaurs because of the spectral contribution of the rings, which is different from that of the main body. Indeed, the centaur color distribution shows a group with red colors and a group of more neutral colors (among which Chiron and Chariklo). If the bimodality is caused by the presence of rings, rings would be very common in centaurs. Recently, Peixinho et al. (2012) have proposed that the color bimodality is also present in small TNOs. If the color bi-modality and the presence of rings are related, small TNOs might therefore have rings as well and under this view the origin of the rings would most likely be in the trans-Neptunian region rather than in the JupiterNeptune region where the centaurs currently reside.

\section{Conclusions}

We presented occultation, photometric, and spectroscopic observations that indicate the presence of ring material around Chiron. In particular, the 2011 occultation lightcurve obtained by Ruprecht (2013) shows secondary events having similar characteristics to that recently found for Chariklo, another centaur. From the occultation an approximate diameter for the proposed ring system of $324 \pm 10 \mathrm{~km}$ has been derived and two possible pole orientations determined. We favor the pole solution $\lambda \sim$ $144^{\circ}, \beta \sim 24^{\circ}$ because it can explain the rotational lightcurve amplitude better at several epochs and can explain the overall historic photometric behavior of Chiron (its absolute magnitude versus time). Also, the changing orientation of the proposed ring system is consistent with the observed variability in the waterice spectral features. Even though the occultation evidence alone might not seem conclusive enough, the other lines of evidence presented in this paper and the similarities with Chariklo are sufficient to make a strong case for the existence of a ring system in Chiron. On the other hand, alternative scenarios, such as cometary jets or a cometary shell for the 2011 occultation features, cannot explain all the observations. That there can be two ring systems in the two largest centaurs might indicate that rings could be common in centaurs and perhaps even in small TNOs, especially if the color bimodality has something to do with the presence of rings. During the revision phase of this paper, the referee pointed out that a paper on the 2011 stellar occultation was submitted by Ruprecht et al. (2015), and a draft was kindly provided to us by the authors. We refer the reader to that publication for the most updated information on that occultation. Their timing remains the same as in Ruprecht (2013), therefore our calculations do not need to be changed.

Acknowledgements. We are grateful to the CAHA and CASLEO staffs. This research is partially based on observations collected at the Centro Astronómico Hispano Alemán (CAHA) at Calar Alto, operated jointly by the Max-Planck Institut fur Astronomie and the Instituto de Astrofísica de Andalucía (CSIC). This research was also partially based on observations carried out at the Complejo Astronómico El Leoncito, operated under agreement between the Consejo Nacional de Investigaciones Científicas y Técnicas de la República Argentina and the National Universities of La Plata, Córdoba, and San Juan. Funding from Spanish grant AYA-2011-30106-CO2-O1 is acknowledged, as is the Proyecto de Excelencia de la Junta de Andalucía, J.A. 2012-FQM1776 and FEDER funds. R.D. acknowledges the support of MINECO for his Ramon y Cajal Contract. A.A.C. thanks the FAPERJ and CNPq for financial support through diverse grants. We are grateful to an anonymous referee for helping us to improve the paper.

\section{References}

Altenhoff, W. J., \& Stumpff, P. 1995, A\&A, 293, L41

Alvarez-Candal, A., Ortiz, J. L., Morales, N., et al. 2014, A\&A, 571, A48

Belskaya, I. N., Bagnulo, S., Barucci, M. A., et al. 2010, Icarus, 210, 472

Binzel, R. P., Farinella, P., Zappala, V., \& Cellino, A. 1989, in Asteroids II, eds.

R. P. Binzel, T. Gehrels, \& M. S. Matthews, 416

Braga-Ribas, F., Sicardy, B., Ortiz, J. L., et al. 2013, ApJ, 773, 26

Braga-Ribas, F., Sicardy, B., Ortiz, J. L., et al. 2014, Nature, 508, 72

Bus, S. J., Bowell, E., Harris, A. W., \& Hewitt, A. V. 1989, Icarus, 77, 223

Bus, S. J., Buie, M. W., Schleicher, D. G., et al. 1996, Icarus, 123, 478 
Bus, S. J., A'Hearn, M. F., Bowell, E., \& Stern, S. A. 2001, Icarus, 150, 94 Campins, H., Telesco, C. M., Osip, D. J., et al. 1994, AJ, 108, 2318

Cikota, S., Ortiz, J. L., Cikota, A., Morales, N., \& Tancredi, G. 2014, A\&A, 562, A94

Duffard, R., Lazzaro, D., Pinto, S., et al. 2002, Icarus, 160, 44

Duffard, R., Pinilla-Alonso, N., Ortiz, J. L., et al. 2014a, A\&A, 568, A79

Duffard, R., Pinilla-Alonso, N., Santos-Sanz, P., et al. 2014b, A\&A, 564, A92

Elliot, J. L., Olkin, C. B., Dunham, E. W., et al. 1995, Nature, 373, 46

Elliot, J. L., Ates, A., Babcock, B. A., et al. 2003, Nature, 424, 165

Elliot, J. L., Person, M. J., Gulbis, A. A. S., et al. 2007, AJ, 134, 1

Elliot, J. L., Person, M. J., Zuluaga, C. A., et al. 2010, Nature, 465, 897

Fernández, Y. R., Jewitt, D. C., \& Sheppard, S. S. 2002, AJ, 123, 1050

Fornasier, S., Lellouch, E., Müller, T., et al. 2013, A\&A, 555, A15

Foster, M. J., Green, S. F., McBride, N., \& Davies, J. K. 1999, Icarus, 141, 408

Groussin, O., Lamy, P., \& Jorda, L. 2004, A\&A, 413, 1163

Lamy, P. L., Gruen, E., \& Perrin, J. M. 1987, A\&A, 187, 767

Lazzaro, D., Florczak, M. A., Angeli, C. A., et al. 1997, Planet. Space Sci., 45, 1607

Lebofsky, L. A., Tholen, D. J., Rieke, G. H., \& Lebofsky, M. J. 1984, Icarus, 60, 532

Levison, H. F., Duncan, M. J., Zahnle, K., Holman, M., \& Dones, L. 2000, Icarus, 143,415

Licandro, J., di Fabrizio, L., Pinilla-Alonso, N., de León, J., \& Oliva, E. 2006, A\&A, 457,329

Luu, J. X., \& Jewitt, D. C. 1990, AJ, 100, 913

Luu, J. X., Jewitt, D. C., \& Trujillo, C. 2000, ApJ, 531, L151

Marcialis, R. L., \& Buratti, B. J. 1993, Icarus, 104, 234
Meech, K. J., \& Belton, M. J. S. 1989, IAU Circ., 4770, 1

Meech, K. J., \& Belton, M. J. S. 1990, AJ, 100, 1323

Noll, K. S., Grundy, W. M., Chiang, E. I., Margot, J., \& Kern, S. D. 2008, in Binaries in the Kuiper Belt, eds. M. A. Barucci, H. Boehnhardt, D. P. Cruikshank, \& A. Morbidelli, 345

Ortiz, J. L., Sicardy, B., Braga-Ribas, F., et al. 2012, Nature, 491, 566

Pasachoff, J. M., Souza, S. P., Babcock, B. A., et al. 2005, AJ, 129, 1718

Peixinho, N., Doressoundiram, A., Delsanti, A., et al. 2003, A\&A, 410, L29

Peixinho, N., Delsanti, A., Guilbert-Lepoutre, A., Gafeira, R., \& Lacerda, P. 2012, A\&A, 546, A86

Person, M. J., Elliot, J. L., Gulbis, A. A. S., et al. 2008, AJ, 136, 1510

Romon-Martin, J., Delahodde, C., Barucci, M. A., de Bergh, C., \& Peixinho, N. 2003, A\&A, 400, 369

Ruprecht, J. D. 2013, in Master thesis of the Massachusetts Institute of Technology, Department of Earth, Atmospheric, and Planetary Sciences http://hdl. handle. net/1721.1/82301

Ruprecht, J. D., Bosh, A. S., Person, M. J., et al. 2013, AAS/Division, Planet. Sci. Meet. Abstr., 45, 414.07

Ruprecht, J. D., Bosh, A. S., Person, M. J., et al. 2015, Icarus, 252, 271

Sánchez-Lavega, A., Wesley, A., Orton, G., et al. 2010, ApJ, 715, L155

Sicardy, B., Colas, F., Maquet, L., et al. 2010, BAAS, 42, 993

Sicardy, B., Ortiz, J. L., Assafin, M., et al. 2011, Nature, 478, 493

Tegler, S. C., Romanishin, W., Consolmagno, G. J., et al. 2005, Icarus, 175, 390

Thirouin, A., Ortiz, J. L., Campo Bagatin, A., et al. 2012, MNRAS, 424, 3156

Timerson, B., Brooks, J., Conard, S., et al. 2013, Planet. Space Sci., 87, 78

Young, L. A., Stern, S. A., Weaver, H. A., et al. 2008, Space Sci. Rev., 140, 93 